\section{How useful are cross-sectional data from surveys of dental caries?}

Burt BA: How useful are cross-sectional data from surveys of dental caries? Community Dent Oral Epidemiol 1997; 25: 36-41. (C) Munksgaard, 1997

Abstract - Surveys are projects involving systematic data collection without a specific hypothesis to be tested and hence without a specific research design. This paper reviews their uses, and some of the issues involved with measuring dental caries in surveys. The principal benefits of surveys are in (a) monitoring trends in oral disease when the surveys are repeated periodically; and (b) giving dental health a visibility it might otherwise not get among policy-makers. On the other hand, they are of limited use in determining treatment needs for a population, evaluating treatment outcomes, and evaluating prevention programs. Some major issues in caries surveys today include difficulties with the DMF index; the use of exclusively visual versus visual-tactile criteria; "hidden" caries; and the appropriate role for early, non-cavitated carious lesions. The DMF index suffers from its mixing of disease and treatment, and more research is needed to determine the most appropriate role for exclusively visual criteria in surveys. Trade-offs, such as weighing the benefits of exclusively visual criteria against the probable greater difficulty in finding "hidden" caries, have not been determined. Inclusion of non-cavitated lesions in a survey will increase its cost. Organizers should therefore be clear before the survey on how this additional information will be used to justify the additional expense.

\section{Brian A. Burt}

Program in Dental Public Health, Department of Epidemiology, School of Public Health, The University of Michigan, Ann Arbor, Michigan, USA

Key words: surveys; dental caries; epidemiology; cross-sectional

Brian Burt, Department of Epidemiology, School of Public Health, The University of Michigan, Ann Arbor, MI 48109-2029, USA

Accepted for publication 10 July 1996
Epidemiology is usually classified as analytical or descriptive, although there is considerable overlap between these categories. Analytical epidemiology means the use of specific research designs (case-control, cohort, case-cohort, retrospective cohort, and others) to test hypotheses to (a) quantify risk in a search for causal relations; (b) assess outcomes of preventive or treatment procedures; or (c) define the natural history of disease. Clinical trials are a specific design to test the efficacy of an agent or regimen. They are classified as analytical epidemiology, though sometimes put into their own category of experimental epidemiology.

I consider the term descriptive epidemiology to be synonymous with surveys, meaning data collected without a specific hypothesis to be tested and hence without a specific research design. The purpose of a survey is to "de- scribe" the oral health status of a population. (Even though hypotheses can be tested from a survey dataset, the data themselves are collected without hypothesis-testing as the prime intention.) Examples of survey data include the Global Oral Data Bank of the World Health Organization (WHO), most of which have been collected under WHO's Pathfinder protocol (1); surveys of nationally representative population samples carried out periodically by government agencies (2-7); and surveys which exploit special programmatic circumstances, such as those in the Child Dental Service in Denmark $(8,9)$ and those conducted by the British Association for the Study of Community Dentistry (BASCD) $(10,11)$.

Surveys absorb a lot of time, energy, and expense, so it is appropriate to ask periodically how useful they are, and whether we can modify our protocols to improve their utility and efficiency. An obvious response is to say "it depends on what the survey is for", and so this paper first looks critically at the uses of survey data involving caries from clinical examinations, and then at the methods of collecting them. Since the emphasis is on cross-sectional caries data, sociodental indicators or other forms of questionnaire-derived measures will not be considered.

\section{How survey data are used}

There are five reasons usually given for why surveys are carried out; these are shown in Table 1. All can be grouped under the general heading of planning and policy development.

Monitoring trends in oral health and disease. When national surveys are repeated periodically under generally similar conditions, broad oral health trends 
Table 1. Uses of survey data

- Monitoring trends in health and disease

- Policy development

- Program evaluation

- Assessment of dental needs

- Providing visibility for dental issues

over time can be estimated. One example is the finding that adults are keeping their teeth more than they used to (12, 13). Results of a national survey should be generalizable to the whole country, and if the sampling design permits they can be generalized to a regional level as well. In a big country, however, sampling becomes too complicated and too expensive to permit generalization to local levels.

Any one national survey, regardless of how elegant its design and conduct, has only limited use without other data to provide a basis for comparison. Provided the sampling desig., so permits, a single survey can show how oral health varies by geographic region, social class, or by race or ethnic group. However, differences between age-cohorts in a national survey, such as in the degree of edentulousness, reflect cohort effects rather than trends. It can never be assumed that today's 30-year-olds will look like today's 70-year-olds in 40 years' time.

The thinking behind WHO's development of the Pathfinder survey protocol was that policy development should be based on trends in oral health rather than on the static results of a single survey. WHO noted that many countries could not economically justify the mounting of expensive surveys with representative samples and carefully controlled data collection, so it devised its approach for the quick and inexpensive collection of oral health data. The protocol is intended to be repeated periodically to assess trends, and it is assumed that the results are valid enough to support national policy decisions (1). While data collected under the Pathfinder protocol are not always validated by more precisely collected information (14), the main issue is whether they are valid enough for the intended administrative purpose. The greatest threat to the validity of Pathfinder data is likely to come from sampling bias.

Policy development. Survey data can be used in policy development to some extent, though there are limitations. In general, the utility of survey data in policy development becomes closer as the jurisdictional level becomes smaller (i.e. the data/policy link becomes less close as it moves from cities and counties to states, provinces, or regions, and eventually to national level). The larger and more diverse the nation, the more likely this is to be true. Examples of the successful use of survey data to develop policy include Scotland, which has used the rich bank of survey data now available in the United Kingdom to establish the Oral Health Strategy for Scotland. Western Australia, geographically large but with a relatively small population, modified both preventive and restorative policies in its school dental service after assessment of the oral health and service data it collects routinely. A number of American states switched their primary preventive focus from fluoride mouthrinsing to sealant application after statewide surveys showed most carious lesions to be in pits and fissures.

Smaller countries with extensive school dental services can fit the model of local jurisdictions. For example, Denmark has used its data to justify moving away from mass procedures like fluoride mouthrinsing toward health promotion on an individual basis (9), and New Zealand redirected its restorative and preventive policies following the first International Collaborative Study of Dental Care Systems (15) in the late 1970 s.

Program evaluation. Survey data are also often used to evaluate programs, though there are clear limitations to this application; the principle that association does not show cause-and-effect needs to be remembered here. To illustrate, the introduction of salt fluoridation into France and Mexico was accompanied by large-scale surveys to establish baselines $(6,16)$. If future caries levels turn out to be lower in those countries, much of the credit will likely be given to salt fluoridation. However, a survey is not a randomized clinical trial and such inferences need to be made with caution. Survey data in program evaluation are subject to the ecological fallacy, i.e. there is no assurance that the people using the agent of interest are the ones in whom the benefit is found, and survey data usually cannot rule out confounding factors. Future improvements in caries status in France and Mexico may or may not be due to salt fluoridation (the issue here not being salt fluoridation per se but that survey data alone are insufficient to support a clear conclusion). If future surveys fail to show a reduction in caries experience, however, it will be reasonable to assume that the salt fluoridation program has not been effective. What this means is that survey data cannot legitimately be used to reject a null hypothesis for program evaluation; the risk of Type I error is too high. The success of particular programs can only be inferred from survey data, though the more localized the survey and the program then the more plausible is the inference.

Assessing treatment needs. Although this is a frequently given reason for conducting surveys (1), treatment need can at best only be assessed in very broad terms. This is because there is a clear gap between the criteria used in surveys and those applied by dentists for patient care, a gap that was demonstrated years ago (17). Criteria for caries in surveys usually are based on cavitation, but dentists generally intervene at an earlier stage in the carious process. A series of studies from the Dundee group in Scotland demonstrated these differences in a follow-up of participants in national surveys of British children and adults. Not only were tooth surfaces in children, called sound in the survey, filled when the child visited a dentist (18), but many teeth in adults that were noted in the survey as needing treatment were either not restored or were extracted (19). Prosthetic needs predicted from the survey in adults bore little relation to treatment subsequently carried out (20).

A limitation of the DMF index is that it carries the assumption that all filled teeth were carious prior to the filling, but there is now good evidence to show that the variation in dentists' treatment decisions leads to the "F" component of DMF scores overestimating caries experience (21).

Assuring visibility for dental issues. The visibility that oral health acquires through the mere existence of data from a national survey may be the most important of all uses of survey data. This is a rather intangible value, but there is little doubt that dentistry fares better in 
policy debates at national level when there are national survey data on which to base an argument. That reason alone may be quite sufficient to make national surveys worthwhile.

\section{Clinical examination methods for caries in surveys}

Recent issues concerning the conduct of surveys that have arisen from recent research are listed in Table 2.

Difficulties with the DMF index. Caries in surveys is usually recorded by the DMF index, the main advantages of which are that it is conceptually simple, can be modified for particular circumstances, and has been widely accepted for many years. Depending on how criteria for caries are specified, the index can be sensitive enough to demonstrate some fairly fine differences between populations (22). The DMF index also has some deficiencies: the absence of a denominator, which means that DMF values need to be presented in agerelated form to have much meaning; and the previously mentioned problem coming from treatment decisions made by dental practitioners. (This means that since easily the major component of DMF scores in the economically developed countries is "F", most diagnostic decisions have been made by untrained, unstandardized, and unknown examiners.) Other issues were not around when the index was first developed: composite and resin restorations can easily be missed, and we are still uncertain about how best to categorize sealants. Nonetheless, the DMF index is likely to be the basis for caries measurement for some time yet.

Examination method: exclusively visual versus visual tactile criteria. From the time of the first national oral health survey in the United States (23) to the most recent such survey $(24,25)$, caries diagnosis has been based on visual and tactile examination. The diagnostic cri-

Table 2. Current issues in data collection in surveys

- Difficulties with the DMF index

- Examination method: exclusively visual versus visual-tactile criteria

- "Hidden" caries

- Should non-cavitated lesions be recorded in surveys? teria for these surveys include tactile softness or visual enamel opacity as evidence of caries; pits and fissures which catch an explorer but without underlying softness or enamel opacity are specifically excluded (5), just as they have been since the US Public Health Service first used the DMF index (26, 27). In the first national survey of British adults in 1968 (2), the probes were filed down to a tip of $0.5 \mathrm{~mm}$, which feels very blunt indeed. Only if the lesion would admit this probe was it called carious. WHO criteria in the Pathfinder protocol diagnose caries by visual-tactile means, and caries is recorded only when the lesion has a detectably softened floor, undermined enamel, or a softened wall (1).

More recently, it has been concluded that caries diagnosis by exclusively visual means loses little sensitivity when compared to the traditional visual-tactile methods $(28,29)$. These studies, however, were conducted with extracted teeth which were later histologically sectioned; it is not yet clear how the findings apply to the field examination conditions used in surveys. Even so, the exclusively visual approach was recently adopted by BASCD for that organization's periodic oral health surveys (30). Other sensitive methods of diagnosing early caries, such as use of electrical conductance and fiber-optic transillumination, are currently under active study and may find a role in surveys. For now, however, none of these issues has been resolved.

"Hidden" caries. A related issue in the discussion over exclusively visual or visual-tactile examination methods is the recognition of the hidden, or "occult", lesion $(31,32)$. This term refers to the radiographically diagnosed dentinal lesion beneath an apparently intact enamel surface. One study with a clinic population reported that hidden caries was found in $15 \%$ of teeth which had no signs of clinical enamel lesions (31). Hidden caries is a widely recognized phenomenon, though it is not yet well understood. It has led to BASCD, in 1992-93, changing its criteria for occlusal lesions to: "If, in the opinion of a trained examiner, after visual inspection there is a carious lesion into dentine" (33). This criterion brings with it a greater emphasis on tooth-drying, and on extensive examiner training. More research is clearly needed on hid- den caries, especially longitudinal clinical studies in which the long-term fate of these lesions can be better determined. The radiographic requirement for its diagnosis is a major problem because radiographs cannot be used in population surveys. It is also not clear whether hidden caries is a modern phenomenon, perhaps related to fluoride therapy, or whether it has been with us, largely unnoticed, for years.

Non-cavitated carious lesions. Caries has traditionally been recorded in surveys as unequivocal lesions, which means dentinal caries or "D3" lesions (34). For the most part, it still is. This practice goes back a long way for, in the first paper to describe the DMF index, criteria for caries were described as: "... teeth which showed actual, though frequently small, cavities. The lesions recorded are those which are readily found on careful clinical examination" (27). This report also noted: "Pits and fissures in which the explorer caught and which after thorough inspection were not considered definitely carious were noted as separate items and were not counted as caries." There was, however, no further mention in the report of these "sticky fissures" (27), a number of which must have been what today would be classed as "D1" or "D2" lesions (34). At that time, and for a long time afterwards, there were plenty of unequivocal lesions to record, so it is hardly surprising that marginal lesions did not get much attention. (The thrust of the Hagerstown studies, where the DMF index was first given its name, was to publicize the bad state of child dental health and the relative absence of adequate treatment at the time. Most of the original DMF paper in 1938 is devoted to calculating the resources needed to treat the disease found.)

The rationale for recording only dentinal lesions in surveys, and not incipient lesions, is that examiner consistency is easier to achieve at this level, and that it is more important to have data that are consistent across examiners than it is to record every possible lesion. Under any circumstances, survey results underestimate the true situation; some lesions will always be missed because examination conditions are less than ideal and radiographs are usually not taken. It is better, the thinking goes, to accept the results as an underestimate 
and not to complicate that issue with inconsistent data. If the data are consistent, then the internal comparisons (i.e. by geographic region or social class) will still be valid.

Criteria for caries in European research studies (not surveys) have long used ordinal grades, for example in the Tiel-Culemborg fluoridation study in The Netherlands (35), which scored caries in one of four grades. In 1979 , WHO listed criteria for recording caries in three ordinal grades, including noncavitated lesions, in its Guide to Oral Health Epidemiological Investigations (36). This guide was intended to be used by those who needed more rigor than the Basic Methods manual, in its second edition at the time, was intended to provide. These WHO criteria were later employed to assess the impact of different diagnostic thresholds on the level of caries prevalence and severity in a population (34). The results of this and subsequent studies $(37,38)$ showed that inclusion or exclusion of non-cavitated lesions made a considerable difference to the status of the populations studied, since there were typically more noncavitated lesions found than dentinal lesions. In a group of 15-16-year-olds in Göteborg, for example, the mean DFS of 6.4 , found when caries was diagnosed at dentinal level, jumped to 19.4 when the non-cavitated lesions were included. While $9 \%$ were free of dentinal lesions or restorations, only $3 \%$ were found to be completely free of all levels of caries (37).

These traditional criteria were established at a time when knowledge of the natural history of caries was limited. There have been many advances since then, the principal one being that we now understand the reversibility of the early carious lesion. Better knowledge of caries dynamics has changed our approach to restorative and preventive dentistry, and caries is recognized as a disease which can progress throughout life (39). A number of respected voices have stated that non-cavitated lesions should be included in surveys $(30,40)$, so the subject is worthy of serious consideration.

\section{Should we include non-cavitated lesions in surveys?}

In research, the inclusion of non-cavitated lesions has undoubtedly helped our understanding of the natural history of caries $(41,42)$ and how dentists treat it (38). While it is apparent that non-cavitated lesions are more common than cavitated lesions in most populations $(37,38)$, there is little longitudinal information on the fate of these lesions. What little there is indicates that a majority either regress or remain static, and those that do progress generally do so slowly, especially when oral hygiene is $\operatorname{good}(39,43-47)$.

There is a strong case for continuing to include non-cavitated lesions in studies which involve the natural history of caries and its treatment. For descriptive surveys, however, the issue is not so clear-cut. There are some instances where the inclusion of non-cavitated lesions would enhance the value of survey data, and others where the additional cost would not be offset by additional benefits. It may be a benefit, for example, to include non-cavitated lesions in a Pathfinder survey which is being carried out to plan oral health programs. For example, if non-cavitated lesions were six times more common than cavitated in 12-year-old children, then sealants would seem an appropriate response. If they were present in equal numbers, however, it could be concluded that caries progressed rapidly in this population and so fluoride use would be more appropriate (despite this being an inference on a longitudinal phenomenon made from cross-sectional data).

The value of including non-cavitated lesions in national surveys, however, is less apparent. Going back to why surveys are carried out in the first place, they have limited value for assessing treatment need and for program evaluation. Surveys rarely follow population samples longitudinally, so they do not follow the natural history of caries. Nor are surveys useful in evaluating treatment outcomes, other than for demonstrating broad trends. Research to determine which treatments are efficacious and cost-effective are best conducted in studies designed to answer those questions, not in surveys. Surveys' main uses are in monitoring disease trends, and perhaps trends in treatment.

\section{Discussion}

Much of the issue of whether survey data would be enhanced if non-cavitat- ed lesions were included comes down to the philosophical basis for surveys. Is it to study the natural history of disease, or is it to take a pragmatic, clinically oriented view of oral conditions? These are quite different aims, though they can be confused. I repeat that survey data are not appropriate for studying the natural history of disease, though they can stimulate hypotheses and provide support for research findings.

It was stated above that surveys underestimate true conditions because of imperfect examination conditions, but at a deeper level they underestimate truth because our disease-detection ability is never perfect. For example, take the case of a national survey, recording dentinal (D3) lesions, which determined that a certain age-group had a mean $\mathrm{D}=$ 0.9 . If non-cavitated lesions were to be included in this survey, then their mean might be 2.4 after the clinical examinations. If more sensitive diagnostic means such as fiber-optic transillumination or electrical conductance were included this figure might rise to 3.2. If a stereo-microscope could be used even more disease would be found, so the mean might rise to 4.2. And if somehow we could get an electron microscope into the act we could detect the earliest enamel dissolution and raise the mean to 6.0. (The numbers are arbitrarily chosen; the issue is that the more sensitive the detection, the more lesions that will be found.) Given that a number of tooth sites are under active demineralization at any one time, the more meticulous measuring methods might also give different results at different times of day. In addition, if the more meticulous diagnostic procedures record the highest proportion of early lesions that will heal without intervention, it is not easy to say which of these possibilities might be considered the nearest approximation of truth in terms of caries experience. Statements such as "Caries experience of a population will be considerably underestimated if the early signs are ignored" (39) need to be assessed thoughtfully in view of the fact that all surveys, no matter how sophisticated the caries diagnosis may be, are only imperfect estimates of truth.

\section{Conclusions}

Surveys serve a major function in monitoring trends in oral disease when they 
are repeated periodically. They are of limited use in determining treatment needs for a population, evaluating treatment outcomes, and evaluating prevention programs. (They can show where prevention has not worked, but associating declining disease with prevention activities does not demonstrate effectiveness of prevention.) Surveys are important in giving dental health a visibility it might otherwise not get in policy-making circles, an important though usually intangible function.

There still needs to be further research on the use of exclusively visual criteria, rather than the traditional visual-tactile criteria, to determine their appropriateness for surveys. The case for using exclusively visual criteria is plausible, but the trade-offs, such as those related to hidden caries, have not yet been well specified.

If non-cavitated lesions are to be included in a survey, the survey cost will be increased. The additional benefits from inclusion of non-cavitated lesions may not offset these additional costs. Organizers should be clear beforehand, therefore, just how this additional information will be used to make the additional expense worthwhile.

\section{References}

1. World Health Organization. Oral health surveys; basic methods. 3rd edn. Geneva: WHO, 1987.

2. Gray PG, Todd JE, Slack GL, Bulman JS. Adult dental health in England and Wales 1968. London: HMSO, 1970.

3. TODD JE. Children's dental health in England and Wales 1973. London: HMSO, 1975.

4. US Public Health Service, National Center for Health Statistics. Decayed, missing, and filled teeth among youths 12-17 years, United States. DHEW Publ. No. (HRA) 75-1626, Series 11 No. 144. Washington DC: Government Printing Office, 1974.

5. US Public Health Service, National Institute Of Dental Research. Oral health of United States children. NIH Publ. No. 89-2247. Washington DC: NIH, 1989.

6. Cahen PM, Turlot JC, Frank RM, OBRy-Musset AM. National survey of caries prevalence in 6-15-year-old children in France. $J$ Dent Res 1989; 68: 64 68.

7. O'Mullane D, Whelton H. Oral health of Irish adults 1989-90. Dublin: Stationery Office, 1992.

8. Helm S, Helm T. Caries among Danish schoolchildren in birth-cohorts 1950-78.
Community Dent Oral Epidemiol 1990; 18: 66-9.

9. Fris-Hasché E, ed. Child oral health in Denmark. Copenhagen: Copenhagen Univ Press, 1994.

10. Pitts NB, Palmer JD. The dental caries experience of 5-, 12- and 14-year-old children in Great Britain. Surveys coordinated by the British Association for the Study of Community Dentistry in 1991/92, 1992/3 and 1990/91. Community Dent Health 1994; 11: 42-52.

11. Pitts NB, Palmer JD. The dental caries experience of 5-year-old children in Great Britain. Surveys coordinated by the British Association for the Study of Community Dentistry in 1993/94. Community Dent Health 1995; 12: 52-8.

12. Downer MC. Changing trends in dental caries experience in Great Britain. Adv Dent Res 1993; 7: 19-24.

13. VON DER FEHR FR. Caries prevalence in the Nordic countries. Int Dent J 1994; 44: $371-8$.

14. Bourgeois D, Leclerce MH, Barmes D. Evaluation of the World Health Organisation pathfinder methodology for oral health surveys in industrialised countries. Community Dent Health 1992; 9: $381-4$.

15. Arnljot HA, Barmes DE, Cohen LK, Hunter PBV, ShIP II. Oral health care systems; an international collaborative study. London: Quintessence and WHO, 1985.

16. Irigoyen M, Szpunar S. Dental caries status of 12-year-old students in the State of Mexico. Community Dent Oral Epidemiol 1994; 22: 311-4.

17. Pickles TH. The relationship of caries prevalence data and diagnosed treatment needs in a child population. Med Care 1970; 8: 463-73.

18. Nuttall NM, Davies JA. The capability of the 1983 children's dental health survey in Scotland to predict fillings and extractions subsequently undertaken. Community Dent Health 1988; 5: $355-$ 62.

19. Nuttall NM. Capability of a national epidemiological survey to predict general dental service treatment. Community Dent Oral Epidemiol 1983; 11: 296-301.

20. Eddie S, Elderton RJ. Comparison of dental status determined in an epidemiological survey with prosthetic treatment need. Community Dent Oral Epidemiol 1983; 11: 271-7.

21. Bader JD, Shugars DA. Need for change in standards of caries diagnosis epidemiology and health services research perspective. I Dent Educ 1993; 57 : 415-21.

22. Downer MC, BLINKHORN AS, Holt RD, Wright C, Attwood D. Dental caries experience and defects of dental enamel among 12-year-old children in north London, Edinburgh, Glasgow and Dublin. Community Dent Oral Epidemiol 1994; 22: 283-5.

23. US Public Health Service, National Center for Health Statistics. Decayed, missing, and filled teeth in adults, United States, 1960-1962. PHS Publ.
No. 1000, Series 11 No. 23. Washington DC: Government Printing Office, 1967.

24. Kaste LM, Selwitz RH, Oldakowski RJ, Brunelle JA, WinN DM, Brown LJ. Coronal caries in the primary and permanent dentition of children and adolescents 1-17 years of age: United States, 1988-1991. J Dent Res 1996; 75 (Spec Issue): 631-41.

25. Winn DM, Brunelle JA, Selwitz RH, et al. Coronal and root caries in the dentition of adults in the United States, 1988-1991. J Dent Res 1996; 75 (Spec Issue): $642-51$

26. Klein H, Palmer CE. Dental caries in American Indian children. Public Health Bulletin No. 239. Washington DC: Government Printing Office, 1938.

27. Klein H, Palmer CE, Knutson JW. Studies on dental caries. I. Dental status and dental needs of elementary school children. Public Health Rep 1938; 53 : 751-65.

28. Lussi A. Comparison of different methods for the diagnosis of fissure caries without cavitation. Caries Res 1993; 27 . 409-16.

29. Lussi A. Validity of diagnostic and treatment decisions of fissure caries. Caries Res 1991; 25: 296-303

30. PITTS NB. Safeguarding the quality of epidemiological caries data at a time of changing disease patterns and evolving dental services [editorial]. Community Dent Health 1993: 10: 1-9.

31. Weerheijm KL, Gruythuysen RJ, van Amerongen WE. Prevalence of hidden caries. J Dent Child 1992; 59: 408-12.

32. Weerheijm KL, van Amerongen WE, EGgINK CO. The clinical diagnosis of occlusal caries: a problem. J Dent Child 1989; 56: 196-200.

33. Pitts NB. Current methods and criteria for caries diagnosis in Europe. $J$ Dent Educ 1993; 57: 409-14.

34. Pitts NB, Fyffe HE. The effect of varying diagnostic thresholds upon clinical caries data for a low prevalence group. $J$ Dent Res 1988; 67: 592-6.

35. Backer-Dirks O, Houwink B, Kwant GW. The results of $61 / 2$ years of artificial drinking water in The Netherlands: the Tiel-Culemborg experiment. Arch Oral Biol 1961; 5: 284-300.

36. World Health Organization. A guide to oral health epidemiological investigations. Geneva: WHO, 1979.

37. Bjarnason S, Kohler B, Ranggard L. Dental caries in a group of 15 to 16year-olds from Goteborg. Part I. Swed Dent $J$ 1992; 16: 143-9.

38. Ismail AI, Brodeur JM, Gagnon P, et al. Prevalence of non-cavitated and cavitated carious lesions in a random sample of 7-9-year-old schoolchildren in Montreal, Quebec. Community Dent Oral Epidemiol 1992; 20: 250-5.

39. Manji F, Fejerskov O. Dental caries in developing countries in relation to the appropriate use of fluoride. $J$ Dent Res 1990; 69 (Spec Issue): 733-41.

40. Fejerskov O. Strategies in the design of preventive programs. Adv Dent Res 1995; 9: 82-8. 
41. Groeneveld A. Longitudinal study of prevalence of enamel lesions in a fluoridated and non-fluoridated area. Community Dent Oral Epidemiol 1985; 13: 159-63.

42. vON DER Fehr FR, Löe H, Theilade E. Experimental caries in man. Caries Res 1970; 4: 131-48.

43. PitTs NB. Monitoring of caries progression in permanent and primary posterior approximal enamel by bitewing radiography. Community Dent Oral Epidemiol 1983; 11: 228-35.
44. Neilson A, Pitts NB. The clinical behaviour of free smooth surface carious lesions monitored over 2 years in a group of Scottish children. $\mathrm{Br}$ Dent $J$ 1991; 171: 313-8.

45. Berkey CS, Douglass CW, Valachovic RW, Chauncey HH. Longitudinal radiographic analysis of carious lesion progression. Community Dent Oral Epidemiol 1988; 16: 83-90.

46. Dummer PMH, Addy M, Oliver SJ, SHaw WC. Changes in the distribution of decayed and filled tooth surfaces and the progression of approximal caries in children between the ages of 11-12 years and 15-16 years. Br Dent $J$ 1988; 164 : 277-82.

47. Klock B, Emilson CG, Lind SO, Gustavsdotter M, Olhede-Westerlund AM. Prediction of caries activity in children with today's low caries incidence. Community Dent Oral Epidemiol 1989; 17: $285-8$. 
This document is a scanned copy of a printed document. No warranty is given about the accuracy of the copy. Users should refer to the original published version of the material. 